\title{
Factors Influencing Chromosome Movements in Mitosis ${ }^{1)}$
}

\author{
By \\ C. W. Metz
}

Department of Embryology, Carnegie Institution of Washington and Department of Zoology, Johns Hopkins University, Baltimore, Md.

Received April 17, 193.5

The subject of chromosome movements has fascinated cytologists almost from the time mitosis was first discovered, and a great deal of attention has been devoted to it by many of the ablest men who have worked in the field; yet, in spite of all the efforts of these investigators, we are still very much in the dark as to the nature of the forces and activities involved in the process under consideration. This fact in itself should be sufficient to deter any one from attempting to explain mitotic movements at this time. The aim of the present paper, therefore, is simply to consider certain factors which seem to be, or may be, involved in chromosome movements. Furthermore, most of the conclusions or interpretations suggested at this time are to be regarded as tentative. The evidence presented is sound, I believe; but the time has hardly come for trying to discover its final implications.

In the early fundamental work on the mechanism of mitosis by Van Beneden, Heidenhain, Bütschli, Hartog, Boveri, Ralph Lillie and many others-work on which all subsequent progress in this field has been based-there was a natural tendency to interpret chromosome movements on the basis of single principles such as contraction of spindle fibers, electromagnetic attraction and repulsion, currents in the protoplasm, pulsating or oscillating bodies at the poles, etc. And correspondingly there was a strong tendency to attribute the movements to the action of outside agents on the chromosomes, rather than to any activity of the chromosomes themselves.

More recently, however, there has been an increasing tendency to recognize combinations of activities in mitosis and to credit the chromosomes with an active part in bringing about their own movements. Suggestions along this latter line have been made by several investigators, including McClung, Bleier, Schrader and Wilson. I

1) This paper was presented before the American Society of Zoologists, at a symposium on mitosis, in Pittsburgh, Pa., Dec. 27, 1934. It is published in its original form, except for the addition of references and footnotes and the omission of certain illustrations which it is not practicable to reproduce. As noted in the text, the treatment is not intended to be comprehensive, but is confined to a few aspects of the subject and a few lines of evidence. 
will not attempt to review these here, but would merely like to ca attention to the fact that this recent trend of opinion has apparentl accompanied a tendency to lay emphasis on exceptional types 0 characteristics of mitosis as distinguished from the more generalize or typical processes previously dealt with.

These unusual phenomena are particularly illuminating, it seem to me, and probably offer us the best available means of attack o1 the problem under consideration. So I feel that I can do no bette than to confine the present discussion largely to such cases. Sinc the time is limited it is necessary either to take just one or tw features and go into them in detail or else to skim rapidly over severa items. I have chosen the latter alternative because I feel that wt are dealing with a complex process and that to get any general vieu of it we must consider several factors. But even such a hasty treat. ment must be strictly limited-so I am going to confine it largely tc material with which I am personally familiar and to aspects of chromosome behavior in which the chromosomes themselves appear to play an active part. I will deal primarily with conditions in the fungus flies (Sciara) and with four specific topics in connection with chromosome movements. One has to do with the significance of the spindle fibers; another with the function of the "insertion body" or point of spindle fiber attachment; a third with the comparative significance of genic and non-genic components of the chromosomes; and the fourth with the possible role of the chromosome sheath. I can not discuss these topics independently, but will try to consider the evidence in relation to them.

If we review the earlier foundation studies on mitosis, it will be found that one of the principles which seemed to be firmly established long ago was that true mitosis, with segregation of chromosomes, could only take place in a bipolar or multipolar field. Unipolar mitosis could be produced experimentally and could be found in pathological material, but it was never accompanied by normal separation of the chromosomes into two groups. I would like to begin discussion, therefore, with the phenomenon of monocentric mitosis which occurs regularly at the first spermatocyte division in Sciara. I have examined about a dozen species of this genus cytologically and have found essentially the same process throughout ${ }^{2}$. It is normal and constant, at this particular division, and it effects a definite and regular segregation of chromosomes by means of actual movements in opposite directions. In this latter respect it differs from all other cases, so far as I know.

2) See Metz 1926a, 1933, and Metz, Moses and Hoppe 1926. 
The mitosis represents the reduction division. The chromosomes are not united in pairs in prophase, but are apparently distributed at random about the periphery of the nucleus. In late prophase they all become oriented in relation to the single pole and "spindle fibers" may be seen extending from them toward the pole (in fixed material). The chromosomes do not move into any one plane comparable to a metaphase plate, but apparently begin their definitive movements directly from their original positions. Certain ones go toward the pole, and certain others retreat from it (fig. 1).

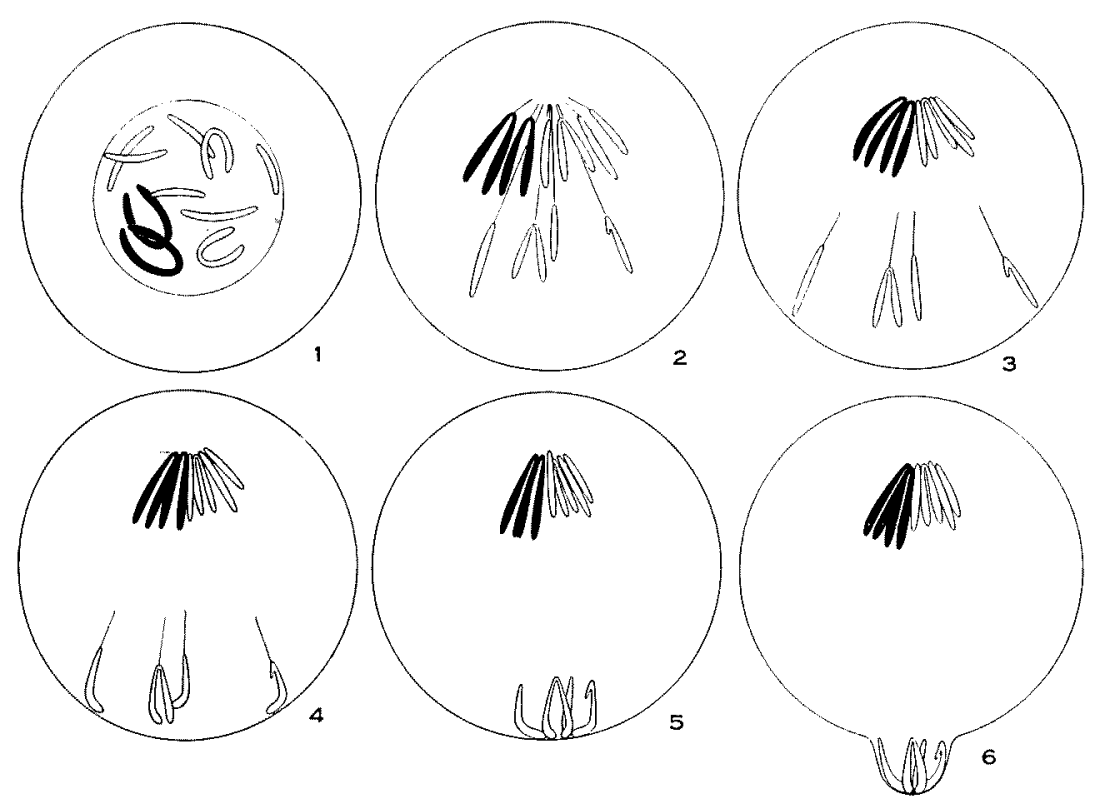

Fig. 1. Diagram illustrating chromosome movements in the monocentric first spermatocyte mitosis in Sciara coprophila Lint. 1. Prophase, showing lack of synaptic association of homologues. 2. Beginning of separation of two groups. The large "limited" chromosomes (represented in black) both go toward the pole; the others separate so that the paternal member of each pair of homologues goes away from the pole and the maternal member toward it. 3 and 4 . Later stages in the movement of the four paternal chromosomes away from the pole. Note their inverted orientation, with spindle fiber attachment hindmost. 5 and 6 . The four chromosomes eventually converge at a point opposite the single pole and are extruded in a polar-body-like process which becomes separated from the spermatocyte. This later degenerates. Compare photographs in 1933 paper.

The former chromosomes exhibit ordinary mitotic behavior, as would be expected. The latter, however, show numerous peculiarities. They move backward, with their free ends in advance and their spindle fiber regions hindmost. They do not go toward any one point or pole, but follow radial paths directly away from the single pole. And in the process they often become greatly attenuated in the region near the spindle fiber attachment. 
It is clear that nothing is pulling these chromosomes away from the pole, because the distal ends are thick and usually curved in various ways-not taut. And it is equally clear that something is acting on the proximal ends (or apex, in the case of the V-shaped member) at the spindle fiber insertion point. This latter agent acts in the direction of the pole, and retards the movement of the chromosomes away from the pole. Since the chromosomes move away in opposition to this agent it seems clear that two opposing forces or activities are involved in this mitosis. One is represented by the spindle fiber and serves, it seems to me, to demonstrate the reality of the so-called spindle fiber activity in mitosis. As to the nature of the activity we are not yet in a position to judge.

The other activity, which in the present case is responsible for the actual movement of the retreating chromosomes, is evidently different from that represented by the spindle fiber. It does not act at any one point on the chromosome, but apparently influences the chromosome as a whole. There is no indication of its being a pulling force. Whatever its nature it seems to transport the chromosomes bodily. This suggests the action of currents or flowing movements in the protoplasm; but as I have noted elsewhere (Metz '33) there are characteristics of the process which do not harmonize with such a conception unless we attribute the movements to an activity of the chromosomes themselves. I have suggested, in the paper just referred to, that the chromosome may be responsible for its own movement by differential alterations in the viscosity of the protoplasm, either anteriorly or posteriorly, or both, and that the chromosome sheath may play a part in the process.

One of the most striking facts about this monocentric mitosis is that it effects a precise and selective segregation of chromosomes, which occurs with an apparently perfect regularity. Leaving out of account, for the moment, the two largest chromosomes shown in the figures (at left in group going to pole) the chromosomes are in pairs. The segregation separates maternal from paternal homologues, causing the former to pass to the pole and the latter away from it, as we have shown by combining genetic and cytological studies ${ }^{3}$. In other words, the direction of movement of two homologues having like sets of genes is determined in some manner here by the sex of the respective parents from which they were derived. This seems to indicate, as I have noted previously, that an influence is exerted on the nongenic material of the chromosomes by the sex of the parent, and that in some manner this determines the direction of movement.

3) See Metz 1926b, 1927, 1928. 
Such considerations immediately raise the question as to whether or not any general distinctions can be observed between the genic and non-genic components of chromosomes in relation to chromosome behavior, and whether or not either type of component can be associated definitely with mitotic or other movements. I cannot go into this question here, but I would like to call attention to the fact that so-called "empty" chromosomes, such as the "Y" chromosome in Drosophila and the "limited" chromosomes in Sciara (the two large chromosomes referred to a moment ago) which apparently contain very few genes, are relatively large

(fig. 2), are deeply staining like the others ${ }^{4)}$ and exhibit essentially the same characteristics of growth, division and mitotic movement as the other chromosomes.

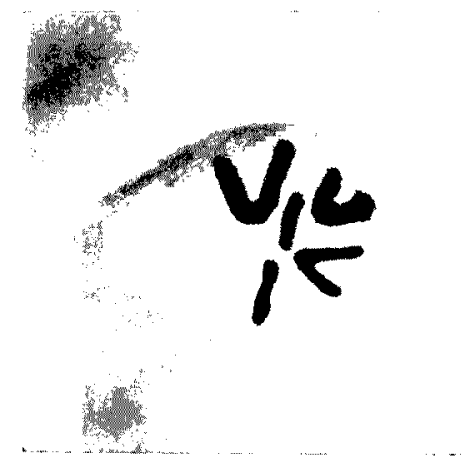

Fig. 2. Photograph of second spermatocyte metaphase group in Sciara pauciseta Felt. Polar view. The precocious chromosome is out of focus and does not show in the picture. The two largest members are the "limited" chromosomes. Note their greater thickness as well as length.

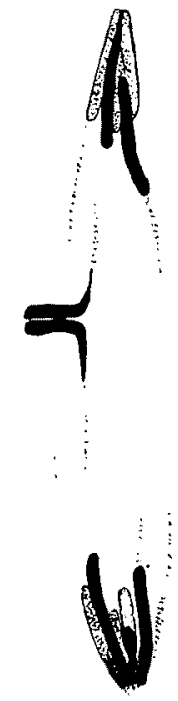

Fig. 3. Elimination of one sex chromosome from somatic nucleus during cleavage in Sciara coprophila Lint. From Du Bois. The chromosome to be eliminated remains at the equator and is thus left lying in the cytoplasm when the two daughter nuclei form at the poles.

Such evidence makes it seem probable that chromosomes possess a non-genic foundation structure which is capable of growth and longitudinal division and which can go through the various mitotic activities characteristic of chromosomes. Presumably it is this material which is responsible for the selective segregation of chromosomes just described in the monocentric mitosis in Sciara.

Another line of evidence which I would like simply to mention, is seen in the processes of chromosome elimination during cleavage

4) See also Heitz' (1933) discussion of heteropycnosis in "empty" regions of chromosomes. 
in Sciara $a^{5} \quad$ Here one chromosome is eliminated which is apparently homologous with one remaining in the cell ${ }^{6)}$. And again selection is apparently based on parentage-maternal and paternal homologues behaving in opposite fashion-indicating that behavior is determined in some way by non-genic materials. But since it is only this particular pair of chromosomes which is involved at this time it seems clear that the genic nature of the chromosome is another factor - unless we assume that each pair differs from all others in its nongenic composition. The latter possibility may be correct, but there is no way of proving it at present, so far as I know.

The general type of interpretation just outlined may be questiond of course-and it may be argued that all the phenomena are due to genic influences, but that in some cases a transient general change in the genes as a whole, or in certain influential genes, is brought about by the sex of a parent, and that this determines the selective type of behavior. There is no evidence for such an interpretation, however, so far as I know, and it goes contrary to the well established principle of genic stability. Likewise it is opposed by the evidence from "empty" chromosomes reviewed above.

Another peculiar type of chromosome behavior in Sciara occurs at the second spermatocyte division. Here one chromosome goes
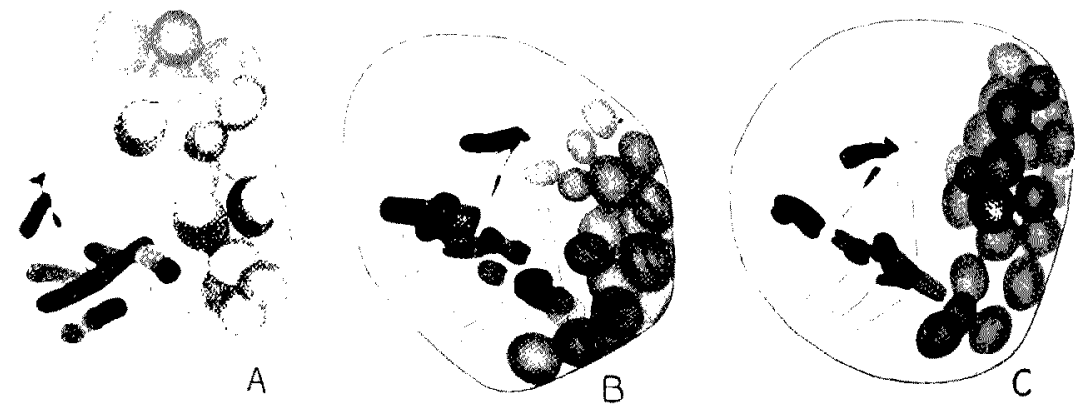

Fig. 4. Second spermatocyte metaphases, side view, from Sciara coprophila Lint. Camera lucida drawings, showing the precocious chromosome at the pole and the other chromosomes at the equator of the spindle. The chromosomes are all split ready for division. The two daughter halves of the precocious chromosome have spindle fibers extending in opposite directions, and the one half is distorted by the "pull" toward the opposite pole. The spherical bodies at one side in each case are chondriosomes. (See also Metz, Moses and Hoppe 1926, figs. 20-24 and 28-43). Spindle fibers schematized in the drawing. They are very faint in the preparation, due to use of Gilson's fixative.

5) See Du Bois 1932, 1933.

6) See Du Bois, loc. cit., and Metz 1931, 1934a.

The elimination referred to here is the second elimination in Sciara (fig. 3). It involves the sex chromosomes. The earlier one involves other chromosomes. The process differs in the two sexes, but the difference in behavior between maternal and paternal chromosomes is evident in both. 
precociously to one pole in advance of the others (fig. 4). The process is constant and regular and always involves the same chromosome. There is no obvious reason why this chromosome should behave in this fashion. It is split in prophase, and the evidence all indicates that the two daughter halves are duplicates of one another. It should, therefore, become oriented on the equatorial plate and send daughter halves to opposite poles like the others. But, instead, the halves go together to the one pole, either in prophase or else in metaphase when the other chromosomes are at the equator.

Before going further with this case I would like to consider very briefly the bearing of the phenomena on some of the general theories concerning the mechanism of mitosis. Several investigators have supported the theory that the anaphase migration of chromosomes is brought about by a system of currents in the spindle. It seems clear that such a system could not cause the regular movement of this one particular chromosome to one pole in the fashion described. Neither could it account for the movements in the monocentric first spermatocyte mitosis described a few minutes ago. Another interpretation of anaphase movement-and a very carefully developed one -is that of Bèlar, based on the idea of growth of the interzonal region of the spindle after autonomous separation of daughter halves has taken place. Yet, clearly, the present case cannot thus be interpreted, for the precocious chromosome goes to the pole before any interzonal spindle comes into existence.

The same objection applies to Bleier's hypothesis based on anaphase movements of the "paragenoplastin" or spindle substance. Likewise, the phenomena cannot be explained on the other principle of Bleier's hypothesis-namely, repulsion between separating halves of a chromosome-for the two halves go together to the same pole. Indeed, the evidence argues against any interpretation based on repulsion between sister chromosomes, or almost any other type of repulsion. Even a repulsion between a chromosome and one of the poles seems to be ruled out by the fact that the precocious chromosome moves toward the pole which forms first. It seems to me that we are forced, tentatively 'at least, to attribute the movement largely to an activity of the chromosome itself.

A suggestion as to what this activity may be comes from the extraordinary fact that, although both halves of the precocious chromosome go to the one pole, the spindle fiber from one of the halves extends toward the opposite pole-presumably through the full length of the spindle. Furthermore, this latter "fiber" represents an activity which serves to distort the chromosome in the direction of the opposite pole (fig. 4). Since this condition exists 
at metaphase, when the other chromosomes are at the equator, and even in anaphase, it seems clear that if the activity in question represents an interaction between the chromosome and the opposite pole it extends directly through the equatorial plate where the other chromosomes lie. This shows pretty clearly that the influence of a pole is not limited to one half of the spindle.

These phenomena also suggest strongly that the spindle fiber activity is, in part at least, an activity of the chromosome itself, and of course that the activity is localized at the insertion point. If this is admitted it follows without question that the "insertion body" or "attachment body" - not the whole chromosome-is the active agent. (It may, of course, act in conjunction with the centriole.)

It is difficult to avoid the conclusion that there is an accurately adjusted coordination here, either between the two sister insertion bodies themselves, or else between them and the respective poles, or both. In this connection I cannot refrain from a hasty mention of two other striking examples of such coordination. One is seen in Oenothera, where, as is well known, the chromosomes at maturation may form a chain in which alternate chromosomes have spindle fiber connections with opposite poles and ultimately pass toward these poles. Unpublished evidence, which Dr. Ralph E. Cleland kindly permits me to cite, indicates that in prophase several successive chromosomes in a series may all be connected with one pole and

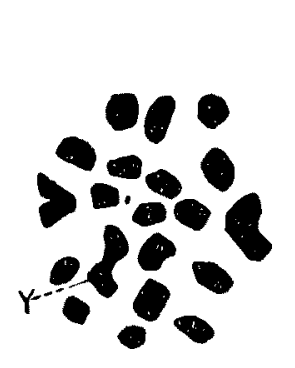

A

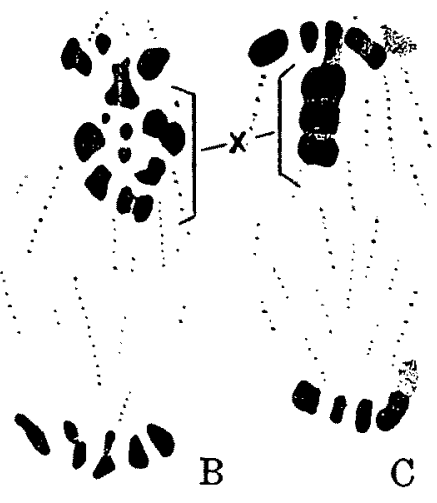

Fig. 5. First spermatocyte division in Contracoecum (Ascaris) incurva. After Goodrich. A. Polar view, metaphase, showing the eight components of the compound $\mathrm{X}$ chromosome lying in the center of the plate. $B$. and $C$. Late anaphases of the same division; side views showing the lagging $\mathrm{X}$-complex going to one pole as a unit, apparently "pulled" by the one component which retains its spindle fiber. The $\mathrm{X}$-complex is seen in face view in $B$. and edge view in $C$. that later alternate ones shift their connections to the opposite pole. The principle of correlation here is obvious.

The other example is that of the compound $X$ chromosome of Ascaris incurva described by Goodrich ('14, '16). Here eight separate chromosomes come together at the first spermatocyte division and then behave as a unit (fig. 5). Furthermore, after they have come 
together all but a certain one lose their spindle fibers and act as if they were inert, so far as mitotic activity is concerned. These seven appear to become attached to the eighth member and to be dragged along by it to the pole. Apparently a stimulus is transmitted from one chromosome to another here after they have come into contact, and this stimulus serves to cause a cessation of mitotic activity in seven of the members. If our present point of view is correct this means a cessation of the activity of the insertion bodies at least. Since it is only these particular chromosomes in the group which behave thus, it seems evident that the behavior is dependent on the specific nature of the individual chromosomes and represents in some manner an activity or reaction of the chromosomes themselves. It also seems to illustrate clearly the coordination already referred to. And the coordination in this case seems to be interchromosomalnot one between the chromosomes and the poles.

This is perhaps as far as we can go at the moment in attempting to interpret the activity represented by the spindle fiber and localized at the insertion point on the chromosome. We are still left, however, with the probability that there is at least one other important activity not accounted for and that this activity is independent of that represented by the spindle fiber. In the monocentric mitosis in Sciara it acts in opposition to the spindle fiber, but in ordinary bipolar mitosis the two presumably act in coordination. This second activity is not localized at any one point on the chromosome, but influences the chromosome as a whole and is apparently determined in some way by the non-genic materials of the chromosomes.

These considerations bring us directly to a discussion of the "chromosome sheath", which has already been mentioned ${ }^{\tau}$. This structure is presumably a gelatinous layer of hyaline material surrounding the chromosome (fig. 6). It has been considered an artifact by some authors: but there are reasonably good grounds, it seems to me, for regarding it as a characteristic

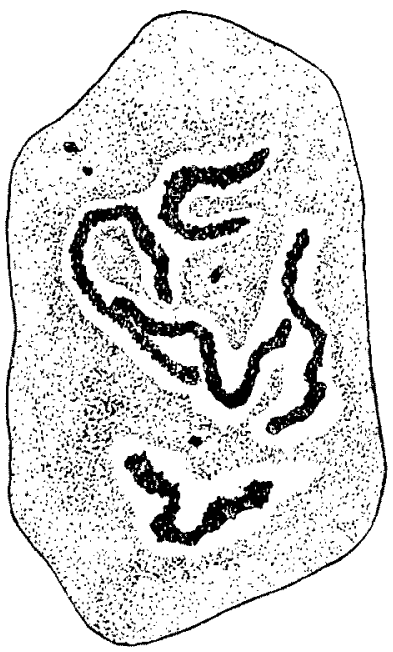

Fig. 6. Portion of nucleus of Lasiopogon bivitattus $\mathrm{Lw}$., in late growth stage of first spermatocyte. The nucleus has collapsed and become a ramifying system of narrow channels. The nuclear membrane comes close to the chromosomes, but not in contact with them-indicating the presence of a "sheath" around each chromosome.

7) For further discussion of the chromosome sheath see Metz and Nonidez 1924, Schrader 1932, Metz $1934 b$ and papers cited in these. 
attribute of chromosomes, and there is also evidence suggesting that it may influence chromosome movements.

There are two points to be considered in connection with the sheath, but one of them I can merely mention. It seems probable, as I have pointed out elsewhere (' $34 b$ ) that the sheath is largely responsible for keeping chromosomes apart, for the spacing of chromosomes in the mitotic figures, for holding sister halves or homologues together during late prophase or metaphase, and for determining many of the mitotic configurations. Indeed I suspect that it accounts in part for the behavior of the compound X chromosome in Ascaris incurva, just mentioned, and that what happens here is that the sheaths fuse and hold the eight chromosomes together, making a sort of pancake. If this is true it suggests that the sheath is under the control of the chromosome itself-and I suspect from other evidence that this is probably the case.

But the feature of particular interest in the present connection is the possible relation of the sheath to ordinary anaphase movements. I have been led to associate the sheath with such movements primarily by study of the retreating chromosomes in the monocentric mitosis described above. In this mitosis it seems clear that each retreating chromosome is surrounded by material of the type just described, which keeps it from contact with chondriosomes and other formed bodies as it passes through the cytoplasm away from the pole. As a result, each chromosome moves off with its surrounding sheath material in the form of a small island which passes bodily through the cytoplasm. It is significant that the retreating chromosomes do not follow any regularly predetermined paths here, but each moves off along any radius from the single pole. Its particular path appears to be determined entirely by its position with respect to the pole at the time the movement starts at the end of prophase.

These facts indicate that the chromosomes are not carried by any system of currents, and they have led me to postulate an activity of the sheath, or of the chromosome through the medium of the sheath, which serves to propel the chromosome and the sheath together away from the pole. A possible means of accomplishing this propulsion, as I have suggested before, is through alterations in the viscosity of the adjacent protoplasm either in front of, or behind, the chromosome, or both. Such a view can be supported, I believe, by considerable evidence both from this case and others, but I cannot go into that here, except to note that the backward movement in this case can be inhibited by cold without stopping the other activities ${ }^{8)}$.

8) See Metz 1933. 
Whether the activity just suggested is an activity of the chromosome or of the sheath material is probably not important at the moment. In any case, if it really occurs, it is probably under the control of the chromosome. Otherwise there is no explanation for the highly selective nature of the segregation here. And if, as the evidence indicates, the selective action is due to the non-genic components of the chromosomes (distinguishing maternal from paternal homologues) it seems reasonable to suspect that these non-genic elements play a part in bringing about the actual movement itself.

These non-genic materials presumably consist of the achromatic matrix of the chromosome and the linin-if these are really two different things. Since the "matrix", in turn, bears a close relationship to the "sheath", and probably gives rise to it in telophase, it may be suggested that matrix and sheath are acting in unison. Such an interpretation could be elaborated at greater length, but the time is hardly ripe for that at present. What is needed now is not more interpretations, but more evidence, and especially more experimental evidence.

\section{Conclusion}

In the brief treatment presented above it has unfortunately been necessary to leave out of account the results of numerous other investigators on the subjects discussed. This has resulted in a narrowly circumscribed presentation. But in general, so far as I know, the other results point in the same direction as those reviewed. It seems increasingly clear that mitotic movements involve complex processes and also that the chromosomes take an active rather than passive part in them. The structure in the chromosome at the base of the spindle fiber (referred to here as the "insertion body") is not only morphologically distinguishable from other structures in the chromosome but probably serves in some manner as an organ of propulsion. It might well be a pulsating body, acting in conjunction with the centriole, or independently. Its activity seems clearly to be correlated with that of other agents.

Another activity is apparently a function of something distributed throughout the entire chromosome instead of localized as is the insertion body. This material seems to be non-genic in nature and is presumably the matrix-sheath substance. It may aid in bringing about chromosome movements by altering the viscosity of the protoplasm in front of and behind the moving chromosome.

Probably other agencies are also acting in the process. Electromagnetic forces may well be operating:' even though it has not yet

9) See, e.g., Kuwada '29 and papers accompanying his. 
been possible definitely to identify their action. Furthermore, it seems probable that agencies may be operative in some organisms which are not present in others-as for example the centrioles in animal cells as distinguished from plant cells.

Considering the fundamental nature of the process of mitosis and the essential uniformity of its results throughout both plants and animals it is only to be expected that a mechanism would exist which would insure its correct operation under widely varying conditions. A single agency could hardly be effective to this extent, and it seems only natural that in most organisms definite insurance should be provided by the presence of additional agencies. One is reminded here of Spemann's principle of "double insurance" in the regulation of development in the embryo. I suspect that in mitosis we have not only double, but probably multiple insurance, and that ultimately several distinct agencies will be identified here, any one of which could perhaps bring about the necessary chromosome movements alone if the others failed to act.

\section{Literature Cited}

Du Bois, A. M. 1982. Elimination of Chromosomes During Cleavage in the Egg of Sciara (Diptera). Proc. Nat. Acad. Sci. 18: 352-356.

- 1933. Chromosome Behavior During Cleavage in the Eggs of Sciara coprophila (Diptera) in Relation to the Problem of Sex Determination. Zeitsch. f. Zellforsch. u. mikr. Anat. 19: 595-614.

Goodrich, H. B. 1914. The Maturation Divisions in Ascaris incurva. Biol. Bull. 27 : $147-150$.

1916. The Germ Cells in Ascaris incurva. Jour. Exp. Zool. 61-91.

Heitz, E. 1933. U̇ber totale und partielle somatische Heteropyknose, sowie strukturelle Geschlechtschromosomen bei Drosophila funebris. Zeitsch. f. Zellforsch. u. mikr. Anat. 19: 720-742.

Kuwada, X. 1929. Chromosome Arrangement. I. Model Experiments with Floating Magnets and Some Theoretical Considerations on the Problem. Mem. Coll. of Sci., Kyoto Imp. Uni. Ser. B, 4: 200-261.

Metz, C. W. 1926a. An Apparent Case of Monocentric Mitosis in Sciara (Diptera). Science, 63: 190-191.

- 1926b. Genetic Evidence of a Selective Segregation of Chromosomes in Sciara (Diptera) Proc. Nat. Acad. Sci. 12: 690-692.

- 1927. Chromosome Behavior and Genetic Behavior in Sciara (Diptera). II: Genetic Evidence of Selective Segregation in S. coprophila. Zeitsch. f. induk. Abstammungs. u. Verer. 45: 184-200.

- 1928. Genetic Evidence of a Selective Segregation of Chromosomes in a Second Species of Sciara (Diptera). Proc. Nat. Acad. Sci. 14: 140-141.

- 1931. Chromosomal Differences between Germ Cells and Soma in Sciara. Biol. Zentr. 51 : 119-124.

- 1933. Monocentric Mitosis with Segregation of Chromosomes in Sciara and its Bearing on the Mechanism of Mitosis. I. The Normal Monocentric Mitosis. II. Experimental Modification of the Monocentric Mitosis. Biol. Bull. 64 : 333-347.

- 1934a. Evidence Indicating that in Sciara the Sperm Regularly Transmits Two Sister Sex Chromosomes. Proc. Nat. Acad. Sci. 20:31-36. 
Metz, C. W. 1934b. The Role of the "Chromosome Sheath" in Mitosis, and its Possible Relation to Phenomena of Mutation. Proc. Nat. Acad. Sci. 20 : 159-163.

_- Moses, M. S., and Hoppe, E. N. 1926. Chromosome Behavior in Sciara (Dip. tera) I: Chromosome Behavior in the Spermtocyte Divisions. Zeitsch. f. induk. Abstammungs. u. Verer. 42: 237-270.

_ and Nonidez, J. F. 1924. The Behavior of the Nucleus and Chromosomes during Spermatogenesis in the Robber Fly Lasiopogon bivittatus. Biol. Bull., 46: 153-164.

Schrader, Franz 1932. Recent Hypotheses on the Structure of Spindles in the Light of Certain Observations in Hemiptera. Zeitsch. f. wiss. Zool. 142 : 521-539. 